Case report

\title{
How should an infected perinephric haematoma be drained in a tetraplegic patient with baclofen pump implanted in the abdominal wall? - A case report Subramanian Vaidyanathan*1, Bakul M Soni ${ }^{1}$, Peter L Hughes ${ }^{2}$, Gurpreet Singh ${ }^{1}$, John WH Watt ${ }^{1}$, Tun Oo ${ }^{1}$ and Pradipkumar Sett ${ }^{1}$
}

Address: ${ }^{1}$ Regional Spinal Injuries Centre, District General Hospital, Southport, PR8 6PN, UK and 2Department of Radiology, District General Hospital, Southport PR8 6PN, UK

E-mail: Subramanian Vaidyanathan* - vaidyanathansiu@hotmail.com; Bakul M Soni - Bakul.Soni@mail.soh-tr.nwest.nhs.uk; Peter L Hughes - Peter.Hughes@mail.soh-tr.nwest.nhs.uk; Gurpreet Singh - Gurpreet.Singh@mail.soh-tr.nwest.nhs.uk; John WH Watt - John.Watt@mail.soh-tr.nwest.nhs.uk; Tun Oo - drtunoo@hotmail.com; Pradipkumar Sett - Pradip.Sett@mail.sohtr.nwest.nhs.uk

${ }^{*}$ Corresponding author

Published: 10 September 2002

BMC Urology 2002, 2:9

This article is available from: http://www.biomedcentral.com/I47I-2490/2/9
Received: 20 May 2002

Accepted: 10 September 2002

(C) 2002 Vaidyanathan et al; licensee BioMed Central Ltd. This article is published in Open Access: verbatim copying and redistribution of this article are permitted in all media for any non-commercial purpose, provided this notice is preserved along with the article's original URL.

\begin{abstract}
Background: We present a case to illustrate controversies in percutaneous drainage of infected, perinephric haematoma in a tetraplegic patient, who had implantation of baclofen pump in anterior abdominal wall on the same side as perinephric haematoma.

Case presentation: A 56-year-old male with C-4 tetraplegia had undergone implantation of programmable pump in the anterior abdominal wall for intrathecal infusion of baclofen to control spasticity. He developed perinephric haematoma while he was taking warfarin as prophylactic for deep vein thrombosis. Perinephric haematoma became infected with a resistant strain of Pseudomonas aeruginosa, and required percutaneous drainage. Positioning this patient on his abdomen without anaesthesia, for insertion of a catheter from behind, was not a realistic option. Administration of general anaesthesia in this patient in the radiology department would have been hazardous.

Results and Conclusion: Percutaneous drainage was carried out by anterior approach under propofol sedation. The site of entry of percutaneous catheter was close to cephalic end of baclofen pump. By carrying out drainage from anterior approach, and by keeping this catheter for ten weeks, we took a risk of causing infection of the baclofen pump site, and baclofen pump with a resistant strain of Pseudomonas aeruginosa. The alternative method would have been to anaesthetise the patient and position him prone for percutaneous drainage of perinephric collection from behind. This would have ensured that the drainage track was far away from the baclofen pump with minimal risk of infection of baclofen pump, but at the cost of incurring respiratory complications in a tetraplegic subject.
\end{abstract}




\section{Background}

Spasticity is a common problem in the patients with tetraplegia due to cervical spinal cord injury. Spasticity may not be relieved by oral medications in a few patients, who may then be considered for implantation of a programmable pump for continuous intrathecal infusion of baclofen. Spinal cord injury patients who have undergone implantation of a baclofen pump require special care while performing procedures in the ipsilateral kidney. For example, while carrying out extracorporeal lithotripsy of renal calculus, which is on the same side as the baclofen pump, precautions need to be taken in order to decrease the risk of damage by shock waves to baclofen pump and the tube [1]. Similarly, spinal cord injury patients with implanted baclofen pump require special attention when a percutaneous procedure is performed in the ipsilateral kidney or perinephric space in order to prevent infection of the baclofen pump. We present a case to illustrate controversies, which arose in clinical management of a spinal cord injury patient who had implantation of a baclofen pump for control of spasticity. This patient with tetraplegia had been taking warfarin, and developed spontaneous perinephric haematoma. The haematoma became infected with a resistant strain of Pseudomonas aerugionsa. The best approach for drainage of perinephric haemastoma was the subject of debate, as there were advantages and disadvantages with each treatment option.

\section{Case presentation}

A 56-year-old male sustained cervical spinal cord injury and developed C-4 complete tetraplegia in May 2001. He underwent implantation of a programmable pump for intrathecal delivery of baclofen, in the anterior abdominal wall in October 2001. This tetraplegic patient was prescribed warfarin to prevent deep vein thrombosis. He developed a spontaneous, right-sided, perinephric haematoma, which became infected with a resistant strain of Pseudomonas aeruginosa. The haematoma was located on the same side as the baclofen pump. We were unsure of the best approach for insertion of a percutaneous drainage catheter without unduly exposing the patient to the risk of the procedure per se, or risk of infection spreading from the perinephric space to baclofen pump.

The patient with cervical spinal cord injury had painful shoulders. He was also suffering from severe trigeminal neuralgia. He was a high-risk case for inhalation anaesthesia on account of his neurological level, gross obesity, poor venous access and especially so, in the setting of radiology department. Positioning this patient on his abdomen without anaesthetising him, for insertion of a catheter from behind into the perinephric space, was virtually impossible. In the prone position, his voluntary ventilation would have been inadequate. Further, a tube for delivery of baclofen from the pump located in the an-

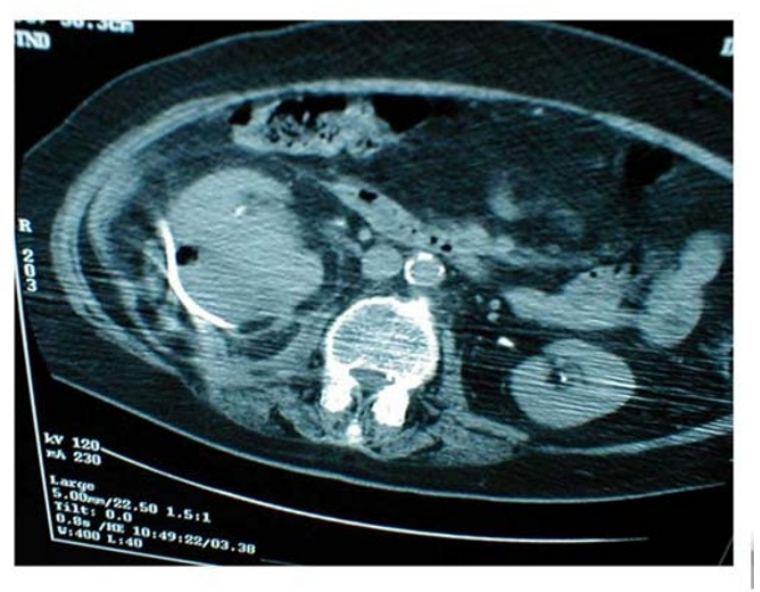

\section{Figure I}

CT of abdomen: Right kidney is enlarged and pushed forwards by the perinephric collection. In contrast to the right kidney, the left kidney is in the normal location and has normal size. Percutaneous catheter, which drains the right pertinephric collection, is seen emerging anteriorly by the side of right kidney.

terior abdominal wall, to the subarachnoid space, coursed around the abdomen to enter the theca posteriorly by the side of L-3 spine.

Since the perinephric collection was presenting in the lumbar region anteriorly, percutaneous drainage was carried out by an anterior approach while the patient was lying supine. The patient received $3 / 4 \mathrm{ml}$ of Cyclimorph-10 (morphine tartrate $10 \mathrm{mg}$ and cyclizine tartrate $50 \mathrm{mg}$ per $\mathrm{ml}$ ) as premedication. Target-controlled sedation with a plasma concentration of propofol of $0.5 \mathrm{microgram} / \mathrm{ml}$ was administered using a Graseby 3400 TCI pump during the radiological procedure, which lasted about ten minutes.

A size 8 Fr. pigtail catheter was inserted through the anterior abdominal wall into right perinephric space while the patient was lying supine. The site of entry of percutaneous catheter was close to the cephalic end of baclofen pump. The position of the catheter as it emerged anteriorly from the right perinephric space is shown in Figures 1 and 2 . The direction of exit of the catheter from the perinephric space is quite different in this patient from that of a traditional approach for drainage of a kidney or perinephric haematoma. In the classical approach for drainage of a perinephric haematoma, the catheter would emerge posteriorly from the perinephric space. The proximity of the drainage catheter as it exits the anterior abdominal wall, to the cephalic end of baclofen pump, is shown in the CT 


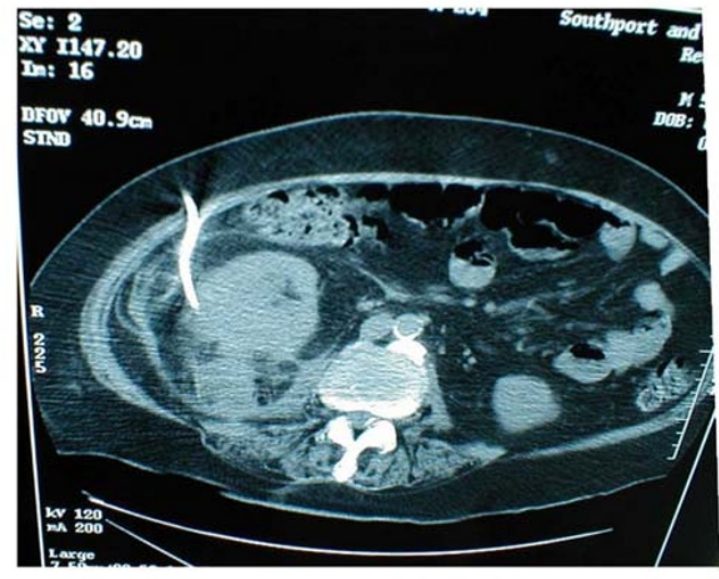

\section{Figure 2}

CT of abdomen: The right kidney is enlarged. There is perinephric collection behind the right kidney. The right kidney is pushed forwards by the perinephric collection. The catheter, which was inserted for drainage of perinephric collection, is seen emerging from the abdominal cavity into the anterior abdominal wall.

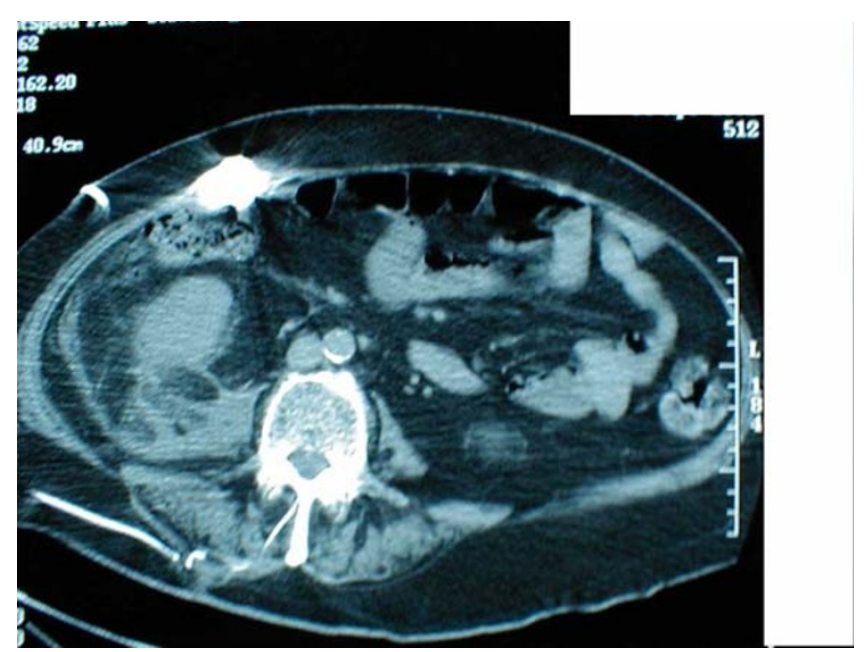

Figure 3

CT of abdomen: The cephalic end of the baclofen pump is seen in the anterior abdominal wall. To the right of the baclofen pump, is seen the pigtail catheter emerging from the anterior abdominal wall. Behind the right kidney is the residual perinephric collection, which appears to have septa. In the posterior abdominal wall just behind the right kidney, is seen the tube which connects the baclofen pump located in the anterior abdominal wall to the intrathecal catheter.

scan of abdomen. (Figure 3). The catheter can be seen exiting to the right of the cephalic end of the baclofen pump.

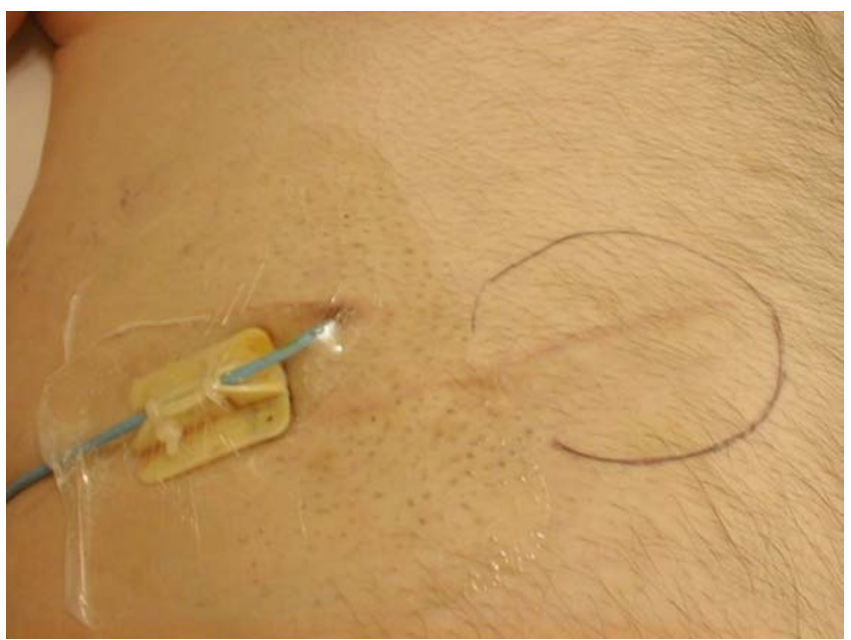

Figure 4

Clinical photograph of the abdomen (close-up view): The surgical scar of implantation of baclofen pump is seen. The pigtail catheter emerges close to the scar. The skin around the pigtail catheter is red and angry-looking. Approximate position of baclofen pump is marked on the skin with a pen.

Clinical photograph of front of abdomen of this tetraplegic patient shows the precise anatomical location of the catheter, as the catheter comes out of the anterior abdominal wall. (Figure 4). A two dimensional relationship of the pigtail catheter draining the perinephric haematoma to the baclofen pump may be appreciated in the scanogram taken during CT of abdomen (Figure 5). The classical way of placing a pigtail catheter from behind for drainage of a kidney is illustrated in figure 6 . This photograph was taken from another tetraplegic subject, who had undergone percutaneous nephrostomy. For placement of a pigtail catheter in the renal pelvis, this tetraplegic subject was positioned on his abdomen and the puncture was made in the lumbar region posteriorly. When figure 6 is compared with figure 4, the differences between the anterior and posterior surgical approaches become very obvious.

Starting from the day of radiological intervention, this patient received colistin, two million units every eight hours, for eleven days. Eighteen days later, he was prescribed amikacin one gram, every 24 hours, for seven days. The skin around the pigtail catheter became slightly inflamed and red. (Figures 4). After he had received colistin and amikacin, a swab, taken from the site of pigtail catheter, showed a heavy growth of Pseudomonas aeruginosa, which was resistant to gentamicin, tazocin and tobramycin. The drainage fluid from the pigtail catheter turned purulent and culture yielded growth of Pseudomonas aeruginosa resistant to gentamicin and tobramycin. When the patient's 


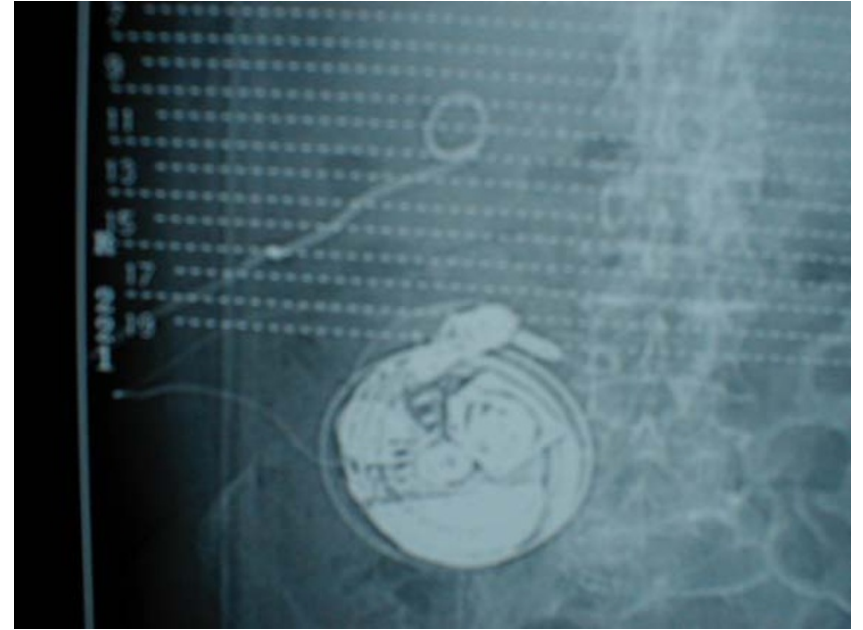

\section{Figure 5}

Scanogram of CT of abdomen shows baclofen pump, which has been placed in the anterior abdominal wall on right side. The tube, which delivers baclofen from the pump into the subarachnoid space, courses around the abdominal wall from the front to the back. A pigtail catheter is seen in the right perinephric space.

clinical condition improved, we considered the option of resiting the catheter from behind. By then, the perinephric collection had become smaller; there were septa within the perinephric space. Therefore, we did not remove the catheter and resite it from the back.

The catheter was removed ten weeks after it had been inserted into the right perinephric space. Prior to removal of the catheter, microbiology of pus, drained by the pigtail catheter, showed a heavy growth of Pseudomonas aeruginosa sensitive to colistin, but resistant to gentamicin and tazocin. A swab taken from the site of entry of the catheter, which was inflamed, showed a heavy growth of Pseudomonas aeruginosa sensitive to colistin and amikacin, but resistant to tazocin, gentamicin and tobramycin. After removal of the catheter, the patient was prescribed amikacin $500 \mathrm{mg}$ intravenously every 12 hours for five days and then, colistin 2 million units 12 hourly for seven days on the basis of the most recent microbiology reports. Twentyfive days later, a sample of urine was sent for microbiology. This showed growth of Pseudomonas aeruginosa sensitive to colistin, but resistant to ciprofloxacin, ceftazidime, imipenem and gentamicin. There has been no clinical evidence of infection of the baclofen pump with Pseudomonas aeruginosa.

\section{Discussion}

In this patient, there were two options for drainage of perinephric haematoma.

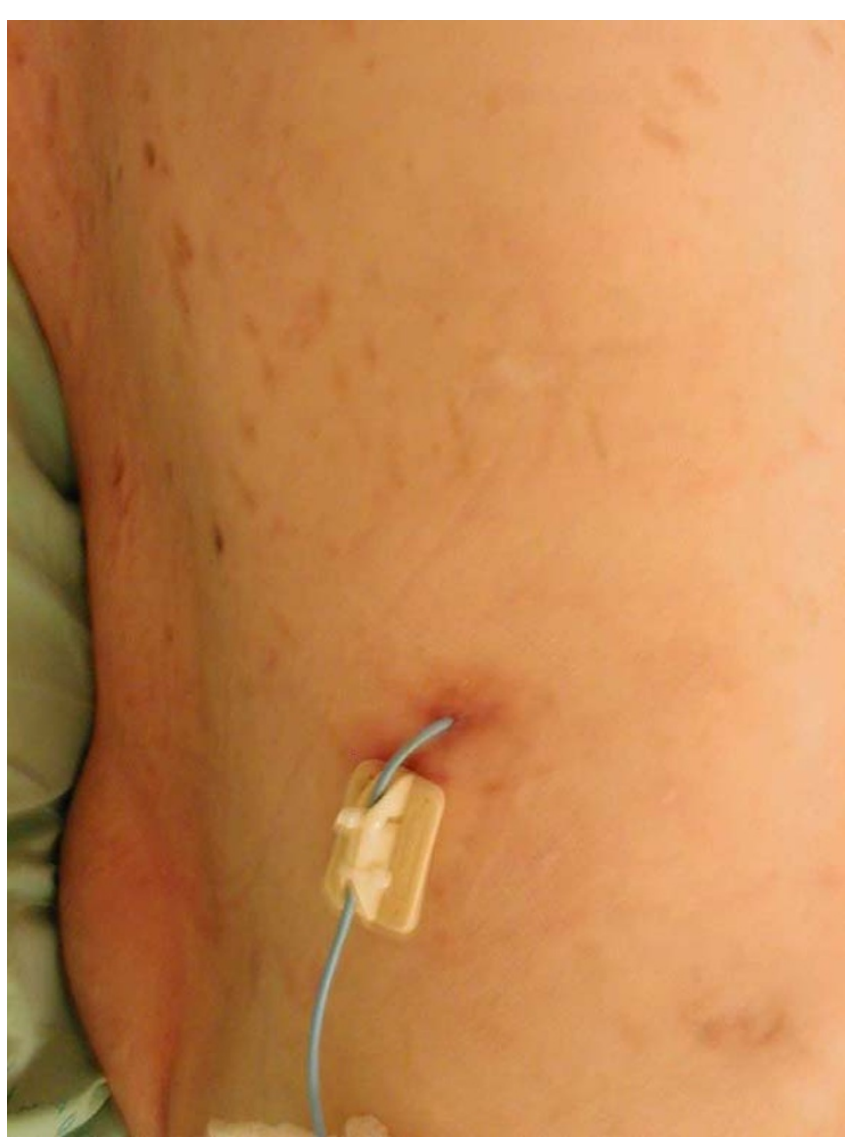

Figure 6

Clinical photograph of another patient with cervical spinal cord injury and tetraplegia: This patient underwent ileal conduit urinary diversion since he had a urinary fistula from the prostatic urethra. Unfortunately, he developed stricture of distal ureters and required percutaneous nephrostomy drainage on both sides. The nephrostomy catheter emerges from the back. The location of the percutaneous nephrostomy catheter represents the traditional approach for draining a kidney or perinephric space, by inserting a catheter from the loin posteriorly. Compare the position of nephrostomy catheter of this patient with figure 4.

\section{- Treatment plan I}

Endotracheal intubation and inhalation anaesthesia followed by turning the patient from supine to prone position. Then the patient would lie on his abdomen and drainage of right perinephric haematoma could be carried out in a traditional way. The point of insertion of a catheter would be located in the lumbar region on the back. The advantages of choosing this treatment option were as follow:

The drainage catheter would be situated far away from the baclofen pump, as the baclofen pump had been placed in the anterior abdominal wall. 
The risk of spread of infection from the perinephric space to baclofen pump would be virtually non-existent.

The disadvantages of this option were the need to anaesthetise a C-4 tetraplegic subject. He could develop respiratory complications after inhalation anaesthesia. He might require prolonged ventilatory support.

\section{- Treatment plan 2}

Drainage of perinephric haematoma could be performed from an anterior approach while the patient was lying supine. The tetraplegic patient would not require general anaesthesia for this procedure. Since the site of exit of the percutaneous catheter would be close to the top end of baclofen pump, there would be a significant risk of spread of infection from the perinephric space to the site where baclofen pump had been implanted. Thus we had to balance the advantages and risks involved in the two options. The risks associated with these two treatment plans are summarised below.

* Risks of treatment plan 1: risks of inhalation anaesthesia, possibility of prolonged ventilation, and post-procedure respiratory complications

* Risks of treatment plan 2: potential risk of infection of baclofen pump site with a resistant strain of Pseudomonas aeruginosa

We chose treatment option 2 because we believed that the risks of inhalation anaesthesia and the occurrence of postprocedure respiratory complications in a C-4 tetraplegic subject were substantial. We were hopeful that with appropriate antibiotic therapy, spread of infection from the perinephric space to the site of implantation of baclofen pump could be prevented. It is likely that some physicians may have decided to anaesthetise the patient, place the patient in a prone position, and then the radiologist would have carried out placement of a pigtail catheter from paraspinal region for drainage of perinephric haematoma. In hindsight, we could have used a more lateral approach for drainage of perinephric haematoma so that the pigtail catheter is more distant to the implanted pump and this would not have required prone positioning. Thus, the treatment plan is open to debate.

\section{Conclusion}

By carrying out drainage of infected perinephric haematoma by an anterior approach under sedation without administering general anaesthesia, and by keeping this catheter in place for ten weeks, we took a risk of causing infection of the baclofen pump site, and baclofen pump with a resistant strain of Pseudomonas aeruginosa. But the patient did not develop any respiratory complications. The alternative method of treatment would have been to anaesthetise the patient and position him prone for percutaneous drainage of perinephric collection from behind. This would have ensured that the drainage track was far away from the baclofen pump with minimal risk of infection of baclofen pump, but at the cost of incurring respiratory complications in a tetraplegic subject.

\section{Authors' contributions}

SV wrote the manuscript. All authors participated in the care of this patient. PH performed percutaneous drainage. JWHW administered target-controlled sedation.

\section{Competing interests}

None

\section{Acknowledgements}

The authors wish to record their gratitude to Dr Ralf Becker, Department of Neurosurgery, Asklepios Kliniken Schildautal, Seesen, Germany for very useful comments. We are grateful to the patient for providing consent for publication of his case in the international journal, BioMed Central, which is free to access anywhere in the world by healthcare professionals and thus helps in dissemination of knowledge. We thank AstraZeneca (Ms Charlotte Lawledge), Pharmacia (Ms Joanne Thomas), and Shire Pharmaceuticals ( $\mathrm{Mr}$ Patrick Tierney) for financial support, which enabled the Regional Spinal Injuries Centre, Southport, United Kingdom to become an institutional member of BioMed Central.

\section{References}

I. Vaidyanathan S, Johnson H, Singh G, Soni BM, Parsons KF: Extra corporeal shock wave lithotripsy of calculi located in lower calyx of left kidney in a spinal cord injury patient who has implantation of baclofen pump in the ipsilateral loin. Spinal Cord 2002, 40:94-95

\section{Pre-publication history}

The pre-publication history for this paper can be accessed here:

http://www.biomedcentral.com/1471-2490/2/9/prepub 